\title{
PET-CT and Occupational Exposure in Oncological Patients
}

\author{
Mikhail Osipov ${ }^{\text {a* }}$, Andrey Vazhenin ${ }^{\mathrm{b}}$, Anna Kuznetsova ${ }^{\mathrm{b}}$, Irina Aksenova ${ }^{\mathrm{b}}$, \\ Daria Vazhenina $^{\mathrm{b}}$, Mikhail Sokolnikov ${ }^{\mathrm{a}}$ \\ ${ }^{a}$ Southern Urals Biophysics Institute, Ozyorskoye Shosse 19, Ozyorsk 456790, Russian Federation. \\ ${ }^{b}$ Chelyabinsk Regional Clinical Center of Oncology and Nuclear Medicine, Bluhera st. 42, Chelyabinsk 454087, Russian Federation.
}

Received 12 March 2020; Accepted 08 May 2020

\begin{abstract}
The paper describes the results of retrospective epidemiological study on low dose effects of diagnostic radiation exposure to humans. The data used in the study has been collected from archives of Chelyabinsk Regional Clinical Center of Oncology and Nuclear Medicine during the project for creating the local electronic database of Ozyorsk patients examined with computed tomography (CTDB). The study group consisted of oncological patients who have received positron-emission computed tomography (PET-CT). All patients in the study group were residents of Ozyorsk - the town in South Ural where the "Mayak" production association, also known as 1st Russian nuclear enterprise is located. The main purpose of the study was to understand whether the number of PET-CT scans, as well as other radiation risk factors influences the mortality in the exposed group. The follow-up period started in 2010 since the first PET-CT scans has been performed, and ended in June, 2019. Radiation dose for oncological patients exposed to PET-CT has been compared with the cumulative dose from occupational exposure. Vital status and cause of death of patients who died to the end of follow-up has been established using the data of National Cancer Registry in part located in the Chelyabinsk Regional Clinical Center of Oncology and Nuclear Medicine. Cancer mortality has been analysed depending on age of patient, sex, number of PET-CT examinations, and presence of occupational exposure, using the logistic regression model. Main radiation risk factors influencing cancer mortality has been established.
\end{abstract}

Keywords: PET; CT; Nuclear Workers; Cancer; Medical Exposure; Radiation Risk.

\section{Introduction}

Positron Emission Tomography (PET) is a modern imaging technique in nuclear medicine developed in last decades. PET is especially helpful in oncology to visualize metabolic processes in malignant tissues. Combined with X-ray Computed Tomography (CT) it substantially enhances the value of diagnostic results [1]. Radiation doses delivered to patients during positron emission computed tomography (PET-CT) are relatively low, but in case of repeated procedures it may lead to significant exposure levels [2]. This is especially important in case when the patient had previously been exposed to ionizing radiation, such as occupational radiation of nuclear workers, since the impact of that radiation exposure on cancer incidence and mortality considered to be significant. Since there are no information on short-term effects of low doses of diagnostic radiation on the human body, nowadays probable (stochastic) effects of this factor is of concern. These effects could be assessed in epidemiological study with long period of follow-up.

However, a feature of long-term study is the presence of time-dependent factors (such as age) which may also affect the risk of outcome analyzed. Therefore, a multivariate analysis is preferable for the study. The objective of the study

\footnotetext{
*Corresponding author: osipov@subi.su

\section{doI http://dx.doi.org/10.28991/SciMedJ-2020-0202-3}

$>$ This is an open access article under the CC-BY license (https://creativecommons.org/licenses/by/4.0/).

(C) Authors retain all copyrights. 
was to assess possible risk factors in the group of oncological patients and understand weather the radiation dose from multiply PET-CT as well as occupational exposure can increase the mortality in the group of oncological patients.

\section{Material and Methods}

\subsection{Material}

The data for the study were obtained during the project on radiation risk assessment of low dose of diagnostic radiation among population of Ozyorsk. As part of the project, the electronic database of Ozyorsk residents examined with computed tomography (CTDB) has been created [3] as a result of joint work of specialists from Southern Urals Biophysics Institute and several medical units of Chelyabinsk region in Russian Federation, including Chelyabinsk Regional Center of Oncology and Nuclear Medicine (CRCONM) where the diagnostic center for positron-emission tomography (PET) is located.

The PET-center of CRCONM contains 2 hybrid positron emission systems combined with multispiral computed tomographs Biograph-40 and Biograph-64. It is important that the PET-center has its own complex for the production of radiopharmaceuticals. Most often, ${ }^{18} \mathrm{~F}-2$-fluoro-2-deoxy-d-glucose (FDG) is used in diagnostics.

The archive protocols analyzed in present study were retrospectively collected in PET-center of CRCONM for Ozyorsk residents examined using Biograph-40 and Biograph-64 in last decade. All patients examined with PET-CT were over 18 year old. Whole-body examinations with radiopharmaceutical (FDG) were delivered to all patients except several cases examined without FDG administration (only CT-mode). The data collection started since the first PET-CT examinations were performed in 2010. The following parameters such as age of the patient, sex, clinical diagnosis, diagnosis at referral, scanner type and number of examinations, administered activity of FDG and other data were recorded. Information on occupational exposure ("Mayak" PA main facilities) has been obtained from "Mayak" workers register [4].

\subsection{Methods}

Retrospective epidemiological study using cohort methodology has been performed. The follow-up period started in 2010 and ended in June of 2019. The cohort included individuals matched the following criteria:

- Residence in Ozyorsk in the period from 2010 to 2019;

- PET examination(s) during the period from 2010 to 2019.

The date of entry to follow-up study was the date of $1^{\text {st }}$ PET examination for cohort members. Since entry, each person followed to the end of follow-up (June, 30, 2019), or the date of death, or the date of lost to follow-up.

Vital status has been updated to June, 2019 for all patients and the cause of death has been established. The death from malignant tumour treated as the event to be analysed.

Patient's effective doses (ED) from CT component were calculated taking into account National Radiation Safety Standards using conversion coefficients [5]. Effective doses from radiopharmaceuticals (FDG) were calculated using conversion coefficients recommended by ICRP 80 [6]. Cumulative ED has been calculated by summing the ED (CT and PET components) in each examination during the whole follow-up period. The ED of occupational exposure of nuclear workers for the entire period of hire at the "Mayak" PA was obtained using film badge dosimetry (FBD) data. Persons without contact to ionizing radiation were considered a zero dose workers. Persons contacted with $\mathrm{Pu}-239$ have been identified and excluded from the analysis.

Since the external exposure to ionizing radiation due to X-ray and internal exposure due to radiopharmaceuticals supposed to be a risk factors, the hypothesis $\mathrm{h}_{1}$ that PET-CT examinations can increase the cancer mortality in the study group has been tested against the hypothesis $\mathrm{h}_{0}$ (no effect of low dose of diagnostic exposure to cancer mortality). Logistic regression realized in STATA statistical package [7] has been applied to test the hypothesis. Odds ratio (OR) was considered as the ratio of probability of an event occurred to the probability of its non-occurrence (1):

$O R=P /(1-P)$

Depending on possible risk factors, a multifactorial analysis was applied using the regression model (2):

$P\left(y_{i} \neq 0 \mid x_{i}\right)=\exp ^{(x i \beta) /(1+\exp (x i \beta))}$

The following parameters $\left(\mathrm{x}_{\mathrm{i}}\right)$ have been used as a regressors:

- $\operatorname{Sex}\left(\mathrm{X}_{1}\right)$,

- Age at $1^{\text {st }}$ PET-CT examination $\left(\mathrm{X}_{2}\right)$,

- Malignant tumor stage $\left(\mathrm{X}_{3}\right)$,

- Occupational exposure to ionizing radiation $\left(\mathrm{X}_{4}\right)$, 
- Repeated PET-CT examinations $\left(\mathrm{X}_{5}\right)$,

Model fitting has been performed using subsequent adding the parameters to the model. Logistic regression model has been tested with different sets of parameters. The quality of fitting has been tested with Chi-square test at $95 \%$ level of significance. The OR coefficients considered to be significant at $\mathrm{p}<0.005$.

\section{Results}

\subsection{Age and Sex Distribution}

651 PET-CT protocols of 347 oncological patients were collected. There are $42.9 \%$ males and $57.1 \%$ females of all ages older 18 in the study group. Age of patients at $1^{\text {st }}$ PET-CT varied from 19 to 91 (average age 57.1 years). Distribution of patients by age categories and sex is shown in Table 1:

Table 1. Distribution of patients by age at $1^{\text {st }}$ PET-CT and sex

\begin{tabular}{cccc}
\hline Age intervals & Male, $\%$ & Female, $\%$ & Both sex, $\%$ \\
\hline $19-29$ & $6.0 \%$ & $0.5 \%$ & $2.9 \%$ \\
$30-39$ & $8.7 \%$ & $7.6 \%$ & $8.1 \%$ \\
$40-49$ & $9.4 \%$ & $17.7 \%$ & $14.1 \%$ \\
$50-59$ & $30.9 \%$ & $23.7 \%$ & $26.8 \%$ \\
$60-69$ & $30.2 \%$ & $37.9 \%$ & $34.6 \%$ \\
$70-79$ & $12.1 \%$ & $9.6 \%$ & $10.7 \%$ \\
$80+$ & $2.7 \%$ & $3.0 \%$ & $2.9 \%$ \\
Total & $100.0 \%$ & $100.0 \%$ & $100.0 \%$ \\
\hline
\end{tabular}

Table 1 shown uneven age and sex distribution of the study group with a predominance of patients older than 50 years. Given this, we used sex and age at $1^{\text {st }}$ PET-CT examination as risk modifiers when modeling.

\subsection{Dose Burden}

During follow-up period $64.6 \%$ of the patients had been examined once, the rest of patients were subject to up to 11 subsequent PET-CT examinations. Average administered FDG activity of $397.5(\min 200.0 ; \max 834.0) \mathrm{mBq}$ per one PET-CT procedure resulted in average effective dose of $7.3(\min 3.0 ; \max 12.8) \mathrm{mSv}$. Average CT dose per one PETCT examination was at 15.7 ( $\min 3.27$; $\max 38.73$ ). According to that, the mean cumulative effective dose for 1 whole body PET-CT examination with average administered FDG activity was $22.2(\min 3.3$; max 71.7$)$ mSv, whereas for patients with repeated PET-CT examinations the mean cumulative effective dose reached $41.2(\min 2.26$; $\max 420.8)$ $\mathrm{mSv}$. Distribution of patients by number of PET-CT examinations and cumulative effective dose from PET and CT components with standard error (in brackets) is shown in Table 2:

Table 2. Mean effective dose (ED) by number of examinations

\begin{tabular}{ccccc}
\hline Number of PET-CT & Proportion, $\%$ & ED $_{\text {FDG }}$ & ED $_{\mathbf{C T}}$ & ED $_{\text {SUM }}$ \\
\hline 1 & 64.6 & $7.3(0.1)$ & $15.7(0.5)$ & $22.2(0.5)$ \\
2 & 19.0 & $14.6(0.3)$ & $30.8(1.3)$ & $46.9(1.4)$ \\
$3-5$ & 12.1 & $25.8(1.4)$ & $61.3(6.3)$ & $92.3(6.5)$ \\
$6-12$ & 4.3 & $56.6(7.0)$ & $131.0(25.8)$ & $180.7(34.6)$ \\
Total & $100 \%$ & $12.6(0.6)$ & $27.8(1.8)$ & $41.2(2.3)$ \\
\hline
\end{tabular}

$21.7 \%$ of patients were nuclear workers hired to main facilities of "Mayak" PA: reactor, radiochemical, plutonium production and auxiliary plants since 1948 to 2000. For those workers, the data on ED from film badge dosimeters were used to compare the mean cumulative dose from external occupational and diagnostic exposure [4]. Among those patients who were nuclear workers $36 \%$ had zero doses of external exposure.

According to the data from Mayak worker registry, 34.7\% of nuclear workers contacted with $\mathrm{Pu}-239$ have been identified. Since exposure to incorporated plutonium is prolonged, we do not provide estimates of the effective dose due to internal exposure for those workers in this study. In order to eliminate the effect of internal exposure to plutonium, these workers were rejected from current analysis. 
The distribution of workers by the number of PET-CT examinations, the mean value of effective dose from diagnostic and occupational exposure (non-zero external doses) with standard error (in brackets), and the proportion of patients occupationally exposed to plutonium-239 is given in the Table 3:

Table 3. Cumulative effective dose (ED) from occupational exposure $(\gamma)$ and PET-CT procedures

\begin{tabular}{ccccc}
\hline Number of PET-CT & Worker, \% & Pu-239 contact, \% & Mean ED $\mathbf{~ , ~ m S v ~}$ & Mean ED PET-CT, mSv \\
\hline 1 & 21.0 & 7.6 & $19.1(8.3)$ & $22.2(0.5)$ \\
2 & 22.7 & 6.0 & $19.5(10.6)$ & $46.9(1.4)$ \\
$3-5$ & 21.4 & 9.5 & $6.4(5.7)$ & $92.3(6.5)$ \\
$6-12$ & 26.7 & 6.7 & $0.4(0.4)$ & $180.7(34.6)$ \\
Total & 21.6 & 7.5 & $11.3(3.6-19.0)$ & $41.2(2.3)$ \\
\hline
\end{tabular}

Mean cumulative effective dose due to repeated PET-CT examinations (i.e. mean additional ED among those patients who had more that one PET-CT examination during the follow-up) was $75.7(64.9-86.4) \mathrm{mSv}$. In the same time, mean cumulative radiation dose as a result of external occupational exposure of nuclear workers with non-zero dose $(11.3 \mathrm{mSv})$ was lower than the dose received by patient during single $(22.2 \mathrm{mSv})$ or even multiply $(41.2 \mathrm{mSv})$ PET-CT examinations, while the ED from external occupational exposure for workers not contacted with $\mathrm{Pu}-239$ was $7.5 \mathrm{mSv}$. The average period of time between the $1^{\text {st }}$ and subsequent PET-CT examinations was 18.2 months (1.5 year).

\subsection{Vital Status and Cause of Death}

To the end of follow-up 51.4\% of examined patients had died at the average age of 60 years. About $4 \%$ of patients from the study cohort were lost to follow-up due to migration from Ozyorsk, and the vital status to the end of followup date was unknown. Distribution of patients by vital status as of June, 2019 is shown in Table 4:

Table 4. Patients by vital status, gender and occurrence of occupational exposure

\begin{tabular}{cccc}
\hline Vital status & Male, $\%$ & Female, $\%$ & Both sex, $\%$ \\
\hline Alive & $39.6 \%$ & $48.5 \%$ & $44.7 \%$ \\
Dead & $57.1 \%$ & $46.9 \%$ & $51.4 \%$ \\
Lost to follow-up & $3.4 \%$ & $4.6 \%$ & $3.9 \%$ \\
Total & $100.0 \%$ & $100.0 \%$ & $100.0 \%$ \\
\hline
\end{tabular}

The period of time from entry to exit from follow-up varied from 0 to 9 years (104 months). Among patients with known vital status $8.8 \%$ has died in the first year of follow-up. Mean period from entry to exit from follow-up was 27 months (2.3 years). Survival function for patients of study group using Kaplan-Meyer statistics is shown in Figure 1:

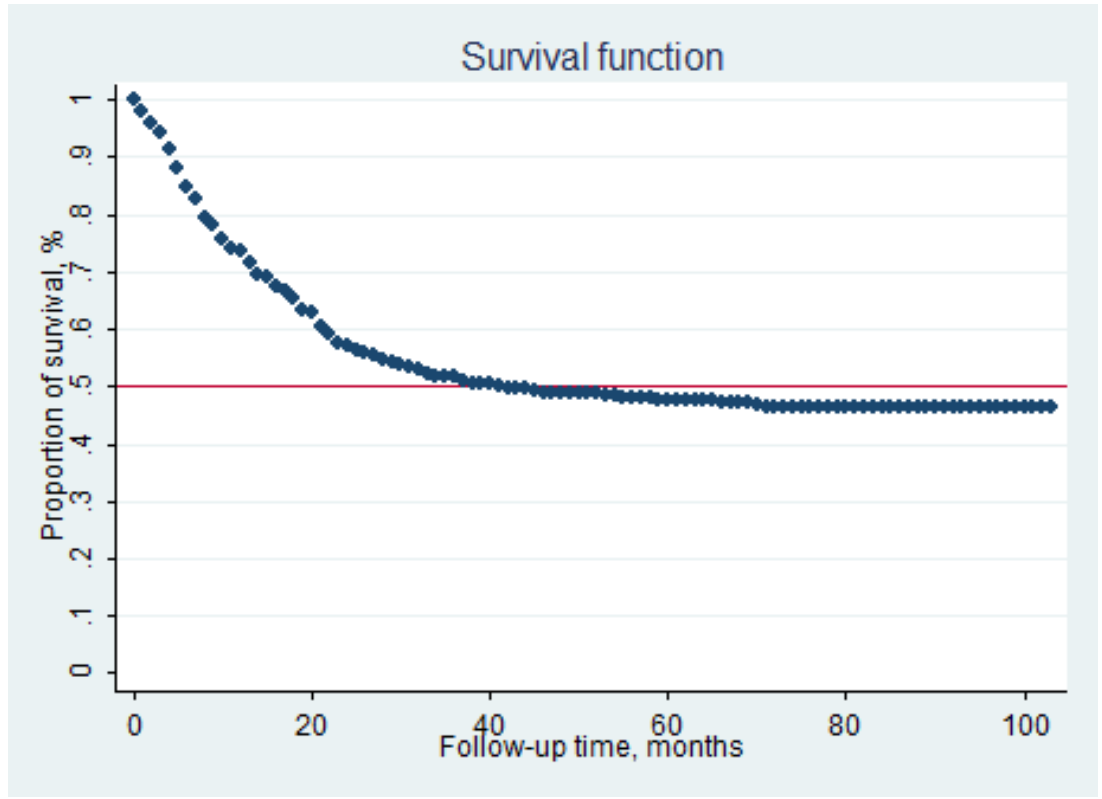

Figure 1. Kaplan-Meyer survival function for oncological patients diagnosed with PET-CT 
On the Figure 1, the mortality in the study group approaches $53.4 \%$ (the patients with unknown vital status has been excluded). The proportion of malignant neoplasms as a cause of death among the study group was $94 \%$. Among patients with confirmed clinical diagnoses of malignant tumours information on stage of malignant tumor established before PET-CT examination has been available in $87.3 \%$ of cases. This parameter has also been used as a regressor.

\subsection{Regression Analysis}

Initial number of patients included in the cohort was 347. The cases with unknown vital status (3.9\%) have been excluded from the analysis. To eliminate the effect of possible internal exposure to incorporated $\mathrm{Pu}-239$, the cases of workers occupationally contacted with plutonium were rejected, thus the number of analyzed cases has been reduced to 272 cases. All parameters were checked for correlation, and the use of parameter as a regressor was allowed in case of correlation coefficient was less than $15 \%$ (weak correlation).

In order to evaluate the effect of external occupational exposure in the study group, 2 sets of parameters were used. The cases with non-zero dose of external occupational exposure were included (Set 1) and excluded (Set 2). Comparison of models with different sets of parameters shown in Table 5:

Table 5. Predictive risk factors and comparison of models

\begin{tabular}{ccccc}
\hline & $\begin{array}{c}\text { Set 1 (external exposure }+ \text { ) } \\
\text { Number of Observed 272 }\end{array}$ & \multicolumn{2}{c}{$\begin{array}{c}\text { Set 2 (external exposure - ) } \\
\text { Number of Observed 246 }\end{array}$} \\
\hline & Prob>Chi ${ }^{2} 0.004$ & Pseudo $\mathrm{R}^{2} 0.04$ & Prob $>\mathrm{Chi}^{2} 0.009$ & Pseudo $\mathrm{R}^{2} 0.04$ \\
\hline Parameter & $\mathrm{OR}$ & $\mathrm{p}$ & $\mathrm{OR}$ & $\mathrm{p}$ \\
$\mathrm{X}_{1}$ & 1.1 & 0.65 & 1.2 & 0.6 \\
$\mathrm{X}_{2}$ & 1.02 & 0.04 & 1.02 & 0.02 \\
$\mathrm{X}_{3}$ & 1.34 & 0.002 & 1.31 & 0.007 \\
$\mathrm{X}_{4}$ & 1.55 & 0.23 & 1.07 & 0.9 \\
$\mathrm{X}_{5}$ & 0.96 & 0.54 & 0.95 & 0.52 \\
\hline
\end{tabular}

Both sets of parameters showed acceptable Chi2 estimates and have been admissible for comparison. Testing of the model showed that $O R$ estimates for parameters $X_{2}$ and $X_{3}$ (age at $1^{\text {st }}$ exposure and stage of malignancy) is statistically significant while the parameters $\mathrm{X}_{1}, \mathrm{X}_{4}$ and $\mathrm{X}_{5}$ failed to reach the significant level.

\section{Discussion}

In this study we have analyzed the data on mortality of oncological patients from Ozyorsk who were exposed to low doses of diagnostic radiation due to PET-CT examinations during the last decade. As far as the authors know, this is the first study on issue of relationship between low doses of diagnostic ionizing radiation from PET-CT and mortality among the group of examined patients. The advantage of the study is using unique source of information the CRCONM PET center archives and "Mayak" worker registry. The methodological strength of research is the use of multivariate analysis: different radiation factors, such as diagnostic and occupational exposure to ionizing radiation, have been analyzed.

It was expected to reveal the impact of age at $1^{\text {st }}$ exposure to PET-CT and stage of the malignant tumour on mortality in the study group. Although radiation exposure is a proven risk factor, there was no statistically significant effect of repeated PET-CT examinations (i.e. additional effective dose of diagnostic exposure due to repeated PET-CT examinations of approximately $50 \mathrm{mSv}$ during the average period of 1.5 years). Therefore, the exposure to a low dose of diagnostic radiation due to PET-CT examinations failed to reveal the impact on mortality in the group of oncological patients. However, relatively short survival period (2.6 years) doesn't reject the possibility of impact of diagnostic radiation on cancer incidence.

As for occupational exposure, the parameter $\mathrm{X}_{4}$ estimate failed to reach the given level of significance in both datasets. Nevertheless, the odds ratio (1.55) obtained for Set1 indicates a possible excess risk, which is described in our previous studies where both solid cancer and leukemia mortality has increased significantly with increasing gamma-ray dose (P 0.001) [8]. At the same time, although the dose of diagnostic exposure could be several times higher than the dose of occupational exposure, an estimate of the OR (0.96) for parameter $\mathrm{X}_{5}$ (the number of PET-CT examinations) indicates that there is no effect on the mortality of this radiation factor. Researchers consider that this phenomenon can be explained by the difference between the scenarios of prolonged occupational exposure and the relatively short diagnostic period during which oncological patients were observed, as well as the severity of the patient's condition. 
At the same time, there are some limitations for this study, which could affect the assessment of the statistical significance of the results. First, it was assumed that the radiation burden of patient due to PET-CT examinations using whole body protocol among different patients gives approximately the same value with small deviations, so the number of procedures is accepted as valid criterion for radiation burden of patient within one procedure. However, for a correct assessment of radiation risk, it is necessary to use the absorbed dose value, which was not calculated in this study due to the complexity of the retrospective restoration of the main parameters (patient size, body weight, scanning parameters, such as Kv, mAs, etc.). In addition, the number of PET-CT examinations may have a positive correlation with the time of observation, therefore, patients who died in the first months after the examination had a little chance to have an additional radiation dose due to PET-CT. However, we observed only a weak correlation ( $\mathrm{r}$ $<15 \%$ ) between these parameters in this study.

Reducing the sample size due to restrictions (unknown vital status and information on contact with plutonium) could decrease the statistical power of the analysis. Also, other unaccounted factors, such as previous X-ray and CT examinations which could increase the patient's dose were probably risk modifiers. However, as for CT, in our previous study in a similar group of oncological patients [9] there was no statistically significant increase in mortality from malignant neoplasms. Since radiotherapy is also associated with exposure to a local dose of ionizing radiation, the data on RT could improve the results of present study. In line with this, further efforts are needed to update information for the studied group using the data from CTDB [3, 10] and CRCONM [11].

\section{Conclusion}

In this study the effect of age at the first PET-CT examination and the stage of the malignant neoplasm on mortality among patients examined with PET-CT have been described. The results also showed that diagnostic exposure due to repeated PET-CT examinations in the group of oncological patients was not associated with changes in mortality. Along with this, the effect of occupational exposure to external gamma-radiation for nuclear workers who has been examined with PET-CT also did not reach the level of statistical significance. Considering this, as well as the limitations described in Chapter 4, further study focusing on collecting additional information on risk factors such as radiotherapy and previous $\mathrm{CT}$ examinations, and the absorbed dose as a measure of radiation risk, is preferable. These results will improve the quality of the computed tomography database of Ozyorsk population (CTDB) which is a unique source of information for conducting a prospective epidemiological study of low-dose effects of diagnostic exposure to humans.

\section{Acknowledgements}

Authors express their sincere gratitude to Sergey Romanov, director of South Ural Institute of Biophysics and member of ICRP, for his help and assistance.

\section{Declaration of Competing Interest}

The authors declare that they have no known competing financial interests or personal relationships that could have appeared to influence the work reported in this paper.

\section{Ethical Approval}

All procedures performed in studies involving human participants were in accordance with the ethical standards of the Chelyabinsk Regional Clinical Center of Oncology and Nuclear Medicine and with the 1964 Helsinki declaration and its later amendments or comparable ethical standards.

\section{References}

[1] Charron, M., Beyer, T., Bohnen, N. N., Kinahan, P. E., Dachille, M., Jerin, J., ... Townsend, D. W. (2000). Image Analysis in Patients with Cancer Studied with a Combined PET and CT Scanner. Clinical Nuclear Medicine, 25(11), 905-910. doi:10.1097/00003072-200011000-00010.

[2] Brix, G., Nekolla, E. A., Borowski, M., \& Noßke, D. (2013). Radiation risk and protection of patients in clinical SPECT/CT. European Journal of Nuclear Medicine and Molecular Imaging, 41(S1), 125-136. doi:10.1007/s00259-013-2543-3

[3] Fomin, E.P., and M.V. Osipov. "Pooled Database Of Ozyorsk Population Exposed To Computed Tomography." Russian Electronic Journal of Radiology 9, no. 2 (2019): 234-239. doi:10.21569/2222-7415-2019-9-2-234-239.

[4] Koshurnikova, N. A., Shilnikova, N. S., Okatenko, P. V., Kreslov, V. V., Bolotnikova, M. G., Sokolnikov, M. E., ... Romanov, S. A. (1999). Characteristics of the Cohort of Workers at the Mayak Nuclear Complex. Radiation Research, 152(4), 352. doi:10.2307/3580220. 
[5] Valentin, J. (1998). Radiation dose to patients from radiopharmaceuticals: (Addendum 2 to ICRP Publication 53) ICRP Publication 80 Approved by the Commission in September 1997. Annals of the ICRP, 28(3), 1-1. doi:10.1016/s01466453(99)00006-8.

[6] Basic Sanitary Rules for Radiation Safety OSPORB-99/2010. SP 2.6.1.25-10 (in Russian). Available online: http://docs.cntd.ru/document/902214068 (accessed on 31 March 2020).

[7] Kohler U., Kreute F. (2005). Data analysis using Stata. USA, Texas: Statapress.

[8] Shilnikova N. S., Preston D. L., Ron E., Gilbert E. S., Vassilenko E. K., Romanov S. A., Kuznetsova I. S., Sokolnikov M. E., Okatenko P. V., Kreslov V. V. Koshurnikova N. A. (2003). Cancer Mortality Risk among Workers at the Mayak Nuclear Complex. Radiation Research 159, 787-798. doi: 10.1667/0033-7587(2003)159[0787:cmrawa]2.0.co;2

[9] Osipov, M. V., Vazhenin, A. V., Domozhirova, A. S., Chernova, O. N., ... Aksenova, I. A. (2019). Computed Tomography As A Risk Factor In Cancer Patients With Professional Radiation Exposure. Russian Electronic Journal of Radiology, 9(1), 142-147. doi:10.21569/2222-7415-2019-9-1-142-147.

[10] Osipov M.V., Sokolnikov M.E. Fomin E.P. (2018). Prospects for using the medical dosimetric register of computed tomography to assess the contribution of medical diagnostic radiation to radiogenic risk. Issues of Radiation Safety, 89 (1): 67 73. (in Russian).

[11] Bray F., Colombet M., Mery L., Piñeros M, Znaor A, Zanetti R, Ferlay J. Cancer Incidence in Five Continents, Vol. XI. Lyon: International Agency for Research on Cancer. Available online: http://ci5.iarc.fr (accessed on 31 March 2020). 